\title{
Avifauna asociada a bosques primarios y secundarios del Parque Nacional Natural Cueva de Los Guácharos, Colombia
}

\author{
Bird species in primary and secondary forests of Cueva de los Guacharos National Park, Colombia
}

\author{
Pablo R. Stevenson ${ }^{1 *}$, Carlos Cortés ${ }^{2}$, Ricardo Reyes ${ }^{2}$, Rosendo Paramero ${ }^{2}$, Jhon F. Uni ${ }^{2}$, Jorge Luis \\ Peña-Núñez ${ }^{3}$, L. Francisco Henao-Diaz ${ }^{1}$
}

\begin{abstract}
Resumen
El Parque Cueva de los Guácharos fue el primer parque nacional declarado en Colombia y, a pesar de su estratégica ubicación, poco se ha publicado sobre la biodiversidad del parque. Este estudio exploró la diversidad de los ensamblajes de aves en diferentes tipos de bosques (andinos, subandinos mixtos y subandinos de roble negro) y el estado de sucesión, que incluye bosques subandinos primarios y secundarios. Para ello, se realizaron puntos de conteo a lo largo de transectos, durante 382 días, entre los años 2005 y 2009 . En este muestreo se reportan 202 especies de aves, representando 39 familias. Las comunidades de aves de los bosques subandinos presentaron mayor diversidad y similitud entre sí, independiente de su estado sucesional. La composición fue diferente en los bosques andinos (dominados por roble blanco, Quercus humboldtii) y de roble negro (dominados por Colombobalanus excelsa, Wettinia fascicularis y Matudaea colombiana). Por ejemplo, la especie dominante en los bosques andinos fue Colaptes rivolii y en los bosques de roble negro fue Zimmerius chrysops. Para los bosques subandinos, tanto primarios como secundarios, se compartieron varias especies en la lista de las más frecuentes: Henicorhina leucophrys, Rupicola peruvianus, Ocreatus underwoodii, Hypopyrrhus pyrohypogaster y Cyanocorax yncas. Este estudio resalta la alta diversidad de los bosques subandinos, incluyendo bosques secundarios cercanos a bosques primarios protegidos. Esperamos que este trabajo sirva de base para el monitoreo futuro de comunidades de avifauna en el parque.
\end{abstract}

Palabras claves: área protegida, comunidades de aves, registros visuales y auditivos, robledales colombianos, tipo de bosque

\begin{abstract}
Cueva de los Guacharos was the first National Park created in Colombia, but few published information is available on its biological diversity. We used point counts along transects for a total of 382 days from 2005 to 2009, to estimate bird species richness and composition in Andean forests, Mixed Sub-Andean forests and Black Oak Sub-Andean forests. In addition, transects were undertaken in both primary and secondary Sub-Andean forests. We report the occurrence of 202 bird species from 39 families. Bird assemblages in mixed primary and secondary Sub-Andean Forest showed the highest species diversity, independent of the successional stage. A non-parametric ordination (based on species abundance) showed three main groupings: Andean forests (plants dominated by white oaks: Quercus humboldtii), Sub-Andean Black Oak forests (dominated by black oaks Colombobalanus excelsa, Wettinia fascicularis, and Matudaea colombiana), and mixed Sub-Andean forests (including both primary and secondary, with a variety of plant species). The most frequently registered bird species in Andean Forest was Colaptes rivolii. Zimmerius chrysops was the dominant species in Black Oak fo-
\end{abstract}

\footnotetext{
1. Departamento de Ciencias Biológicas, Universidad de Los Andes, Bogotá, Colombia.

2. Unidad de Parques Nacionales, Parque Nacional Natural Cueva de Los Guacharos, Palestina, Huila.

3. Corporación Mashiramo, Colombia, Huila.

*Autor para correspondencia: <pstevens@uniandes.edu.co>
} 
rests. In both mixed primary and secondary Sub-Andean forests the composition was similar with several species in the list of the most frequently observed: Henicorhina leucophrys, Rupicola peruvianus, Ocreatus underwoodii, Hypopyrrhus pyrohypogaster and Cyanocorax yncas. This study emphasizes that bird communities in Sub-Andean forests are highly diverse, and secondary forest show similar ensembles to primary forests, when they are protected and located near primary forests. We hope that this study will help to carry out future monitoring programs of bird species in the park.

Keywords: Avian communities, Colombian oak forests, forest type, visual and vocal records, protected area

\section{INTRODUCCIÓN}

Colombia está dentro de los países con mayor diversidad de aves y posiblemente el más rico del mundo con cerca de 1941 especies (Ayerbe-Quiñones, 2018). Gran parte de esta diversidad se encuentra en la zona Andina y el norte del país (Vélez et al., 2021), donde ha ocurrido una alta tasa de destrucción de hábitats naturales (Armenteras et al., 2011; Etter et al., 2006). Como respuesta a la protección de esta preciada biodiversidad, una herramienta clave corresponde al sistema de áreas protegidas del país (Kattan et al., 2006); No obstante, hay importantes vacíos de información en algunas de las áreas protegidas. Por ejemplo, en la recopilación de información de aves para las 25 áreas protegidas de la zona Andina de Colombia (Franco et al., 2007), para nueve de ellas no se logró obtener información publicada. Aunque hay trabajos clásicos para la región (Chapman, 1917), también hay un creciente conocimiento de la distribución de especies en bases de datos nacionales e internacionales (e.g. GBIF, SiB Colombia). Así, los compendios de información sobre avifauna que se conocen de las áreas protegidas son variables, desde listados de especies hasta descripciones cuantitativas por tipo de hábitat (Álvarez et al., 2003; Cadena et al., 1998; Suárez-Sanabria y Cadena, 2014). Para efectos de conservación de poblaciones amenazadas, la información cuantitativa de abundancias relativas por tipo de bosque y acercamientos al recambio de especies entre tipos de bosque (diversidad beta) es relevante para generar planes de acción y realizar monitoreos. Sin embargo, no es común encontrar este tipo de información en los parques nacionales naturales (PNN).

El PNN Cueva de los Guácharos fue la primera área protegida declarada en Colombia y en este se han registrado 296 especies de aves y 62 especies de mamíferos, incluyendo especies amenazadas (Gast y Stevenson, 2020; Parques Nacionales Naturales, 2005). Posee un terreno montañoso que contiene numerosas cuevas y cañones causados por los afluentes del río Suaza. El parque incluye ecosistemas andinos y subandinos que hacen parte de los biomas más diezmados de Colombia (Armenteras et al., 2011), los cuales prestan varios servicios ecosistémicos, incluyendo la regulación y producción de agua por medio de captación de niebla (Cavelier y Goldstein, 1997). Las instalaciones del parque están en los límites entre bosques secundarios y primarios, ya que el origen del parque corresponde a la compra de fincas en las cuales hubo ganadería y explotación de algunas maderas (Parques Nacionales Naturales, 2005). Entre los bosques naturales, una de las principales diferencias a nivel florístico se encuentra influenciada por la elevación, evidenciada en comunidades de plantas distintivas de los bosques andinos (entre 2400 y 2700 m s. n. m.) y los subandinos (debajo de 2400 m s. n. m.) (Parques Nacionales Naturales, 2005). Recientemente se caracterizó la flora de estos ecosistemas a partir de levantamientos de vegetación en 20 parcelas de 0,1 ha (Prada y Stevenson, 2016). Según este estudio, las principales diferencias en flora ocurren porque los bosques andinos primarios $(1=\mathrm{BAP})$ tienen como elemento dominante al roble blanco (Quercus humboldtii), y a nivel estructural son bosques con árboles, en general, de baja altura. Esto contrasta con los bosques subandinos $(2=\mathrm{BS})$, que pueden tener árboles hasta de $40 \mathrm{~m}$ de altura (Henao-Diaz et al., 2012). En este tipo de bosques también se presenta una marcada diferencia florística entre dos tipos de bosque, los bosques primarios mixtos $(2 \mathrm{a}=\mathrm{BSP})$ (en adelante "bosques subandinos primarios") y los bosques primarios dominados por roble negro $(2 \mathrm{~b}=\mathrm{BSRN})$ (Colombobalanus excelsa), la palma Wettinia fascicularis y Matudaea colombiana (en adelante "bosques de roble negro"). Por último, 
la caracterización florística incluye otra división que corresponde a la historia de uso de los bosques, ya que hay tanto bosques subandinos primarios (BSP) como secundarios (BSS).

Las especies con mayor densidad en los levantamientos de bosques primarios subandinos fueron: Wettinia kalbreyeri, Alfaroa colombiana y Hedyosmum cuatrecazanum. Estos bosques se destacan por el alto porte de sus árboles, como Tapirira guianenesis subandina e Incadendron esseri. Dentro de los elementos arbóreos más abundantes en los bosques secundarios se encuentran: Chamaedorea linearis, Saurauia brachybotrys, Nectandra acutifolia y Oreopanax microcephalus. Acá también se resalta la presencia de Heliocarpus americanus y Croton magdalenae, dentro de los árboles de mayor tamaño en bosques secundarios (Prada y Stevenson, 2016).

El PNN Cueva de los Guácharos fue principalmente declarado como zona protegida con el objetivo de resguardar una colonia de aves conocidas como guácharos (Steatornis caripensis) (Franco et al., 2009). No obstante, en la actualidad se considera un lugar clave de conservación para otros elementos de fauna, incluyendo poblaciones de primates en peligro crítico de extinción (Vargas et al., 2014). Adicionalmente, se considera un punto estratégico para aves migratorias y está incluido dentro de las 116 áreas más importantes para la conservación de las aves (AICA) (Franco et al., 2009). Además, es una zona biogeográfica relevante, por ser uno de los puntos más bajos de la cordillera oriental, lo que potencialmente puede haber permitido flujo de individuos a través de las montañas del norte de los Andes (Cadena et al., 2016). Sin embargo, no se tienen registros publicados de su avifauna, ni análisis sobre los patrones de diversidad. El objetivo de este trabajo fue evaluar la riqueza de especies de aves en el PNN Cueva de Los Guácharos, analizar si existen diferencias en la diversidad entre las comunidades de cada tipo de bosque y resaltar algunos elementos importantes de conservación (e.g. presencia de especies con algún grado de amenaza).

Nuestra principal hipótesis de trabajo fue que las comunidades deben estructurarse principalmente por los recursos disponibles, ya que las diferencias en disponibilidad de alimento pueden generar nichos de forrajeo diferentes entre tipos de bosque (BojorgesBaños y López-Mata, 2006; Martínez y Rechberger, 2007). Según esto, esperamos encontrar diferencias en la composición de las comunidades de aves locales. Además, se ha resaltado que las comunidades de aves varían fuertemente en gradientes altitudinales (Kattan et al., 2004; Renjifo et al., 1997) y, por lo tanto, también se espera encontrar diferencias en composición entre bosques subandinos y andinos.

\section{MATERIALES Y MÉTODOS}

\section{Área de estudio}

El Parque Nacional Natural Cueva de Los Guácharos [PNNCG], está ubicado en los departamentos del Huila y Caquetá, Colombia. Su superficie total es de 9000 ha, con alturas entre los 1800 y 3000 m s. n. m. Su precipitación anual es de $3100 \mathrm{~mm}$ con una temperatura anual promedio de $16^{\circ} \mathrm{C}$ (Parques Nacionales Naturales, 2005). La variación en el relieve, su ubicación tropical y las condiciones de alta humedad, le atribuyen a la zona una alta diversidad florística (Prada y Stevenson, 2016). De acuerdo con la geomorfología, dentro del PNNCG se distinguen ocho unidades de paisaje, principalmente en ecosistemas andinos y subandinos. Para cada uno de estos ecosistemas se diferencian tres unidades geomorfológicas, cerros, picos o laderas; sin embargo, esta variación topográfica no se refleja, necesariamente, en la composición florística de los bosques (Prada y Stevenson, 2016).

\section{Registro y análisis de datos}

La Unidad Administrativa Especial del Sistema de $\mathrm{PNN}$, con el fin de conservar la riqueza de flora y fauna del país, estableció un plan de monitoreo en el Parque Cueva de los Guácharos. Dicho plan incluye, entre sus objetivos, obtener información del estado de los valores objeto de conservación en el parque (servicios ecosistémicos, atributos culturales y biodiversidad, incluyendo 14 especies de aves). Por esto se han realizado inventarios de la biodiversidad, pero los métodos de muestreo han variado en el tiempo. En este estudio mostramos la información con los procedimientos que se realizaron inicialmente, 
que corresponden a conteos en puntos a lo largo de transectos o avistamientos a lo largo de ellos. Estos muestreos se ejecutaron durante 382 días, entre los años 2005 a 2009 (incluyendo toma de datos en todos los meses del año y con la posibilidad de registrar tanto especies locales como migratorias). El esfuerzo de muestreo se distribuyó, por tipo de bosque, de la siguiente manera: $2 \%$ bosque primario andino, $7 \%$ primario roble negro, $16 \%$ primario subandino y $76 \%$ secundario subandino. Los puntos de muestreo estaban separados al menos $30 \mathrm{~m}$ entre sí, y el tiempo de permanencia en cada uno fue de $10 \mathrm{~min}$. En total, se instalaron 7 transectos de $300 \mathrm{~m}$ cada uno, en un rango altitudinal de 1700 a $2600 \mathrm{~m}$ s. n. m. y localizados sobre 4 trochas principales (figura 1). Cuando no se utilizaron puntos, sino transectos, la velocidad de recorrido estuvo entre 1 y $2 \mathrm{~km} . \mathrm{h}^{-1}$. Solamente en 316 de los 5770 registros (5\%) se detectó a la misma especie de ave en puntos contiguos, indicando que la probabilidad de no independencia, al comparar con una probabilidad aleatoria, es muy baja $(p<0,001)$.

Con el fin de estimar la diversidad local de los cuatro tipos de bosque muestreados, se calcularon varios índices. Primero, se comparó la riqueza de especies mediante un análisis de rarefacción en $\mathrm{R}$ (R Development Core Team, 2012). Este análisis también permite evaluar la representatividad del muestreo realizado. Posteriormente, se calculó el índice de diversidad alfa entrópico de Shannon con 1000 réplicas por bosque, con el fin de comparar la diversidad de aves. Igualmente, se generaron modelos de distribución sobre curvas de riqueza-dominancia. Los análisis se llevaron a cabo mediante los paquetes vegan (Oksanen et al., 2013) y bootstrap (Tibshirani y Leisch, 2015) en R (R Development Core Team,

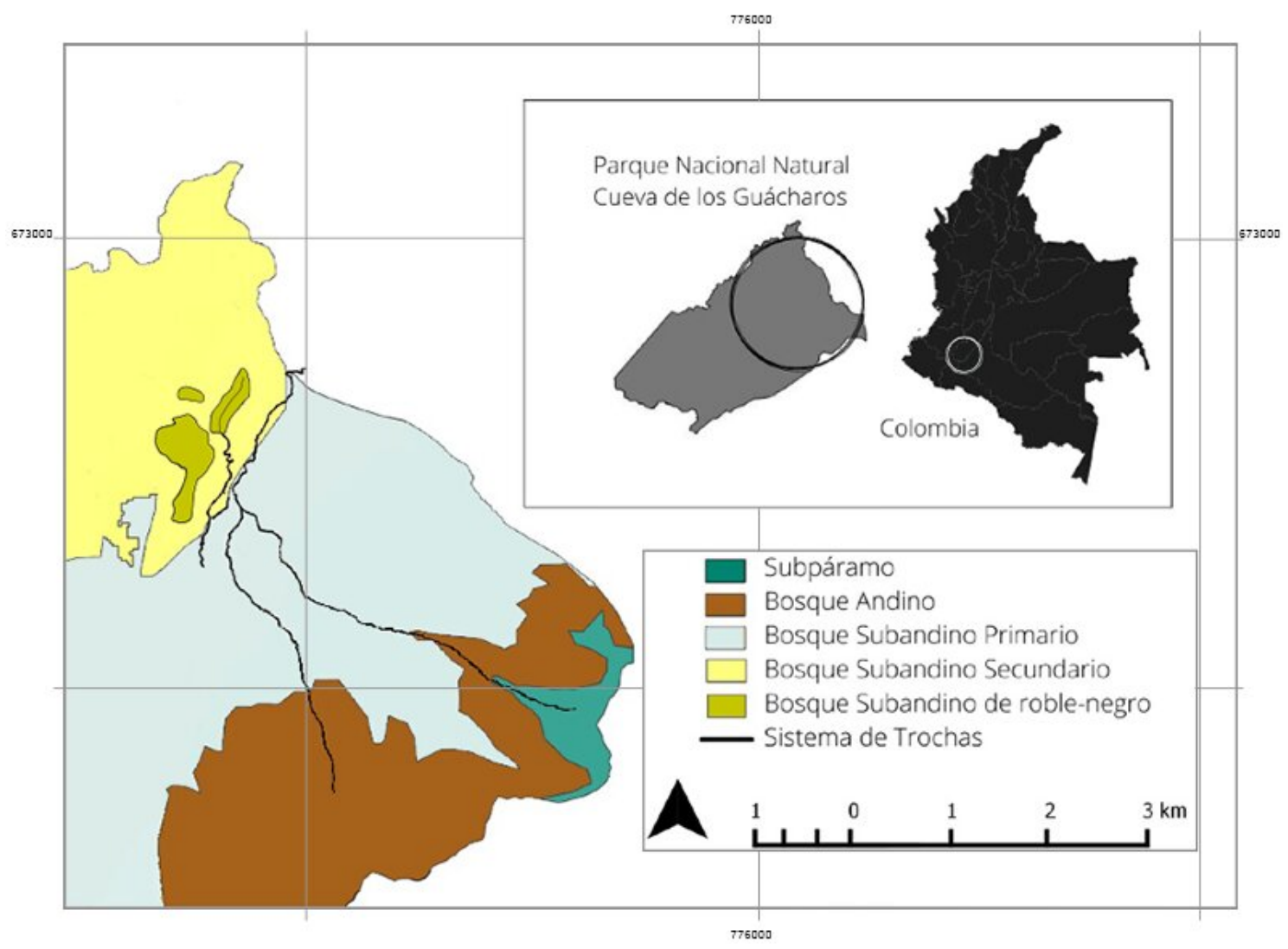

Figura 1. Ubicación de los bosques primarios andinos y primarios y secundarios subandinos en el Parque Nacional Natural Cueva de Los Guacharos, Huila (Colombia). Se indica la localización de las trochas con los transectos y puntos de muestreo para el registro de aves. 
2012). Finalmente, para explorar la diversidad beta o recambio en el tipo de especies de aves entre las comunidades vegetales, se realizó un análisis de escalamiento multidimensional no paramétrico (NMSD). Esta ordenación de similitud se basa en la composición de las comunidades en términos de la frecuencia de registros de las distintas especies en los tipos de bosque, utilizando el índice de similitud de BrayCurtis.

\section{RESULTADOS}

En los muestreos se registró un total de 202 especies, de acuerdo con la nomenclatura de Remsen et al. (2021) (anexo 1), de las cuales 32 de ellas se reportaron en bosque primario andino, 29 en roble negro, 121 en subandino mixto y 185 en secundario subandino. Sin embargo, estos datos no son directamente comparables ya que, por las limitaciones logísticas, el esfuerzo de muestreo fue diferente en cada tipo de bosque. Para efectos comparativos, el análisis de rarefacción para los dos bosques con mayor esfuerzo de muestreo arrojó que el bosque primario contiene ligeramente más especies de aves que el secundario (figura 2). Adicionalmente, el bosque secundario subandino tiene un esfuerzo de muestreo más adecuado que el bosque primario subandino, dado que en este último la curva de acumulación aún tiene tendencia en aumento (figura 2). Por otra parte, el índice entrópico de Shannon mostró diferencias significativas entre los tipos de bosque (Kruskal-Wallis H: 3212 , g. $1 .=3, p<0,001)$ siendo los bosques primarios subandinos y secundarios subandinos los que mostraron una diversidad significativamente mayor, a los bosques andinos y de roble negro (figura 3). Los modelos de diversidad-dominancia mostraron mayor dominancia en los subandinos de roble negro (figura $4)$.

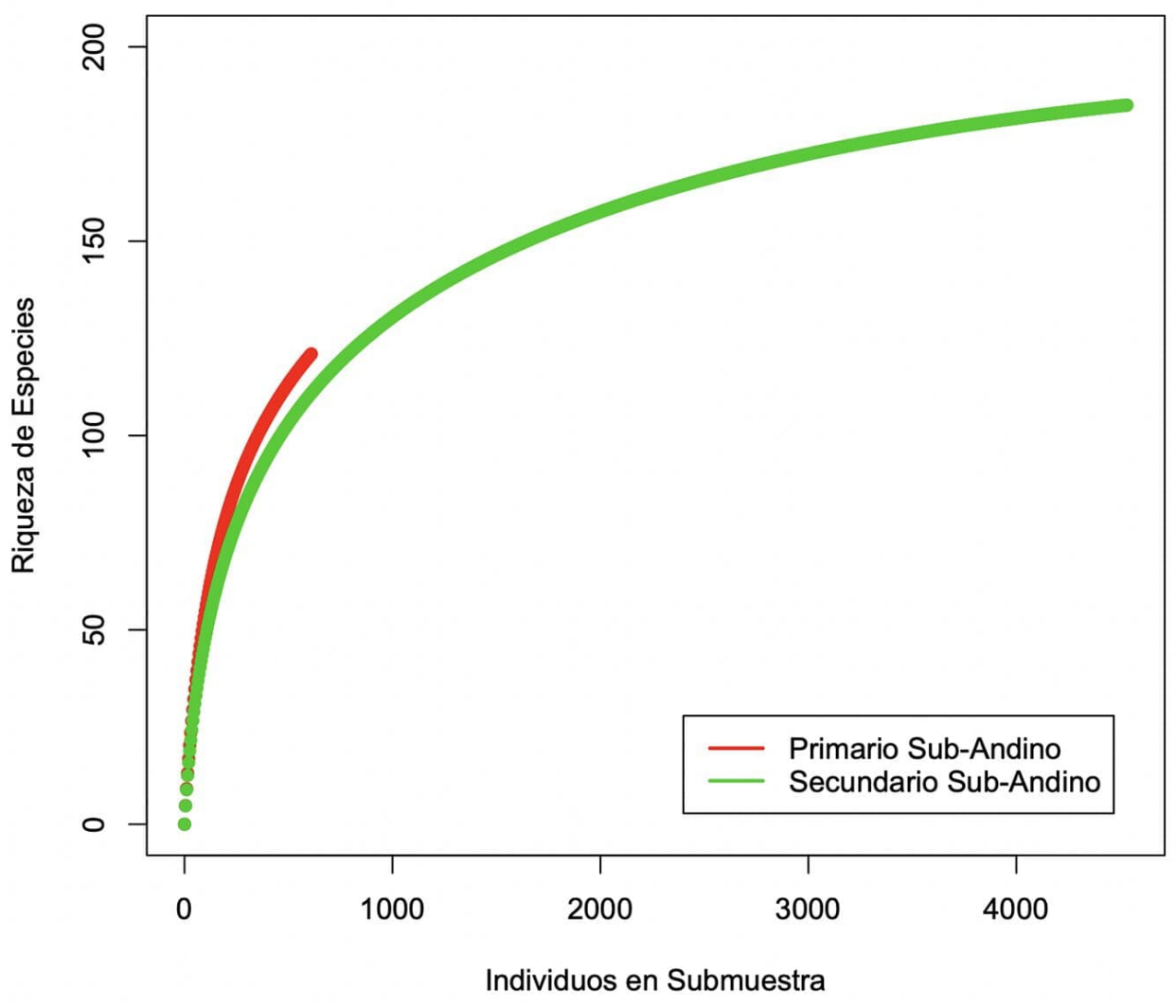

Figura 2. Análisis de rarefacción de la riqueza esperada de especies de aves para bosques primarios y secundarios subandinos en el Parque Nacional Natural Cueva de Los Guacharos, Huila (Colombia). 


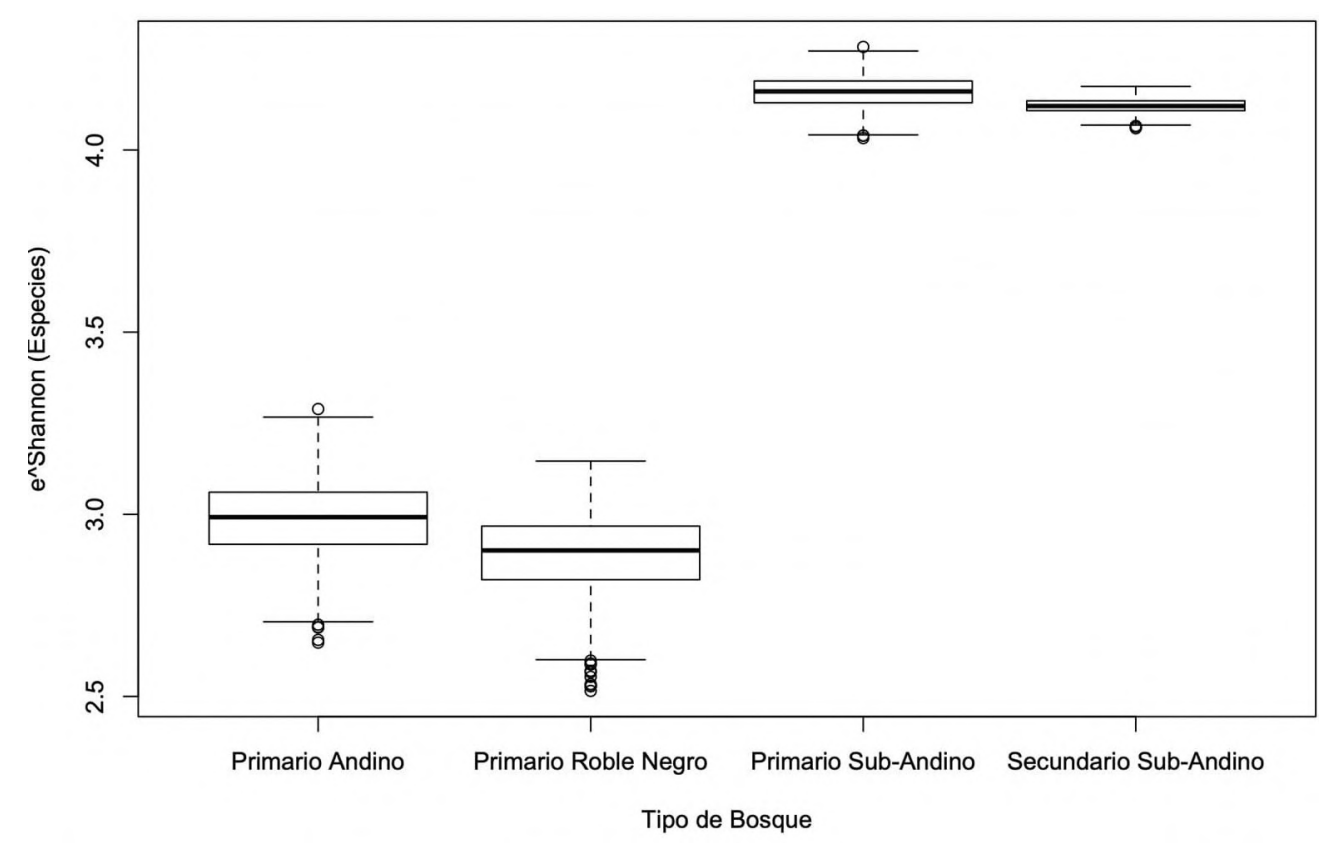

Figura 3. Índice entrópico de Shannon ( $\left.\mathrm{e}^{\mathrm{H}^{\prime}}\right)$ de las comunidades de aves para cada tipo de bosque muestreado en el Parque Nacional Natural Cueva de Los Guácharos. La comparación arrojó diferencias estadísticas significativas en la diversidad de aves (Kruskal-Wallis $\mathrm{H}=3212$, g.l. $=3, p<0,001$, Bootstrap $=1000$ réplicas por tipo de bosque).

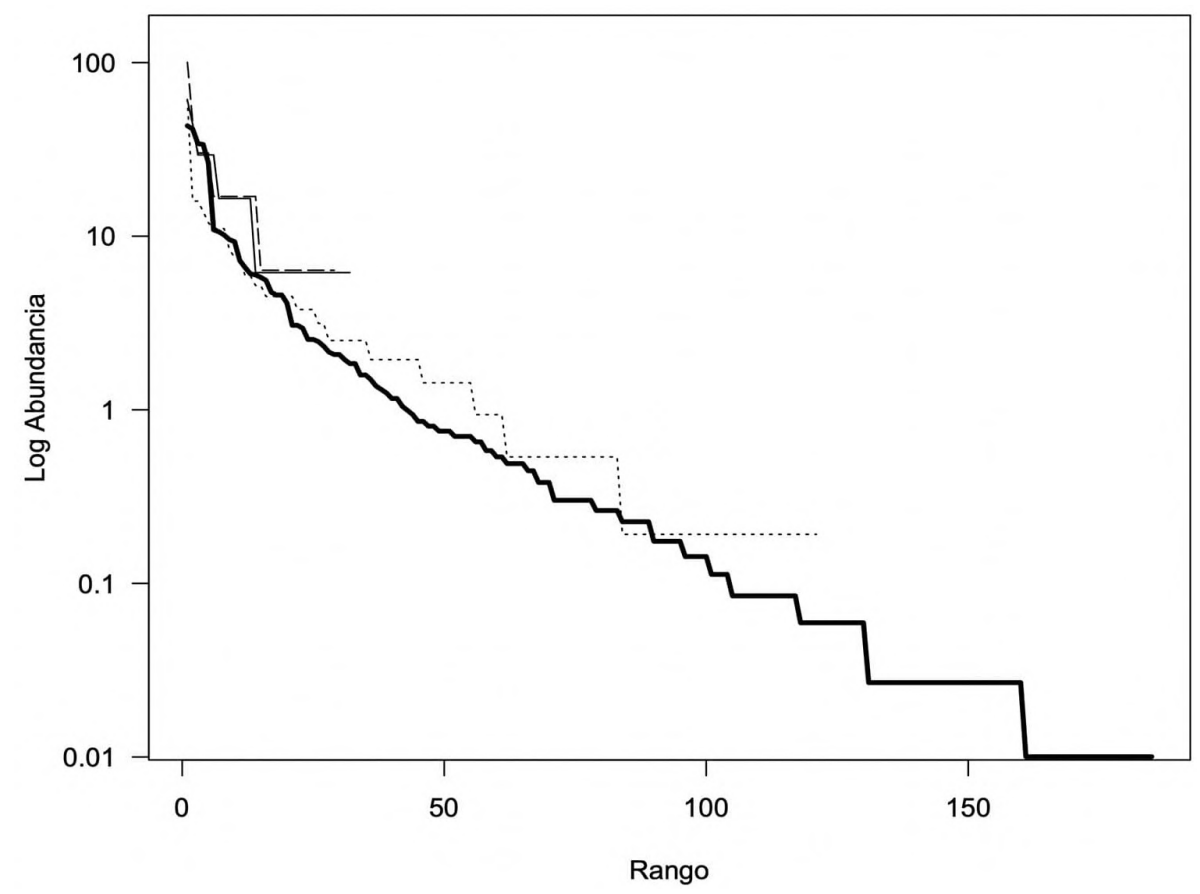

Figura 4. Curvas de riqueza-dominancia de las comunidades de aves para cada tipo de bosque muestreado en el Parque Nacional Natural Cueva de Los Guácharos. Para cada curva se ajustó el mejor modelo de acuerdo con el criterio AIC. Línea continua gruesa: Bosque Secundario Subandino. Mandelbrot, AIC =849,5. Línea punteada: Bosque Primario Subandino. Lognormal, AIC = 387,8. Línea interrumpida: Bosque Primario Roble Negro. Zipf, AIC = 74,8. Línea continua: Bosque Primario Andino. Zipf, AIC = 80,5. 
La ordenación mostró que la composición de especies presenta una mayor similitud entre los bosques primario subandino y secundario subandino (figura 5). Las especies más abundantes en cada tipo de bosque fueron en general diferentes, excepto entre los bosques subandino primario y secundario (tabla 1 ). De hecho, dentro de la lista de las diez especies con más registros, seis especies se comparten entre estos dos tipos de bosque: Henicorhina leucophrys, Rupicola peruvianus, Ocreatus underwoodii, Hypopyrrhus pyrohypogaster, Kleinothraupis atropileus y Cyanocorax yncas.

$\mathrm{Al}$ contrastar los registros de especies con las bases de datos de especies en peligro de la Unión Internacional para la Conservación de la Naturaleza (UICN) para Colombia y globalmente, se registró el tinamú gigante (Tinamus osgoodi) y el loro orejiamarillo (Ognorhynchus icterotis) como especies en peligro (EN); igualmente, se registraron especies vulnerables (VU) y casi amenazadas (NT) (tabla 2). Aunque estas especies se registraron en varios tipos de bosques, todas ellas tuvieron registros en bosques secundarios subandinos.

Se identificaron 15 especies migratorias, entre las cuales predominaron las especies con migración altitudinal $(n=9)$ y local $(n=8)$. La migración altitudinal se presenta en algunas especies que permanecen todo el año en el país, pero se mueven entre distintas franjas de elevación en respuesta a la disponibilidad de flores y frutos (Naranjo et al., 2012). Por su parte, la migración local, que puede en ocasiones ser transfronteriza y es también un movimiento cíclico dentro de un mismo cinturón latitudinal en respuesta a la disponibilidad de hábitat o a la presencia de recursos abundantes en parches específicos, se da principalmente en loros, aves frugívoras y aves acuáticas (Naranjo et al., 2012).

En cuanto a especies con migración latitudinal se registraron seis, las cuales se reproducen en latitudes templadas de Norteamérica y de Suramérica (anexo 2). Estas llegan una vez al año hasta Colombia y permanecen en el país durante varios meses, antes de emprender el regreso a sus sitios de anidación (Naranjo et al., 2012). En la comunidad de aves estudiada se presentan tres especies con migración latitudinal boreal y tres especies con migración austral.

\section{DISCUSIÓN}

En acuerdo con lo esperado, la diversidad de aves si cambió entre tipos de bosque, donde los bosques andinos en elevaciones mayores a $2400 \mathrm{~m}$ s. n. m. resultaron ser menos diversos que los de elevaciones intermedias, como se ha encontrado en otros estudios (Ferreira y Perbiche-Neves 2021; Kattan et al., 2006). Sin embargo, la diversidad de aves de los bosques de roble negro resultó ser mucho menor a lo esperado por su elevación. Esto probablemente se debe a que este tipo de bosque tiene una altísima dominancia de tres especies de plantas y una baja diversidad florística (Prada y Stevenson, 2016). Adicionalmente, dos de las especies de plantas más abundantes $(\mathrm{Co}$ lombobalanus excelsa y Matudaea colombiana) muestran patrones multi-anuales de producción de frutos (Stevenson, observación personal). Esto implica que la oferta de recursos es poco variada, especialmente por la escasez de frutos carnosos y los pocos recursos ocurren muy espaciados en el tiempo. Por esta razón, varias especies de mamíferos y aves muestran densidades muy bajas en este tipo de bosque (Vargas et al., 2014).

Los bosques primarios se componen, en su mayoría, por árboles longevos con una alta riqueza de especies, lo que atrae a varios frugívoros, consumidores primarios y polinizadores (Taki et al., 2013). Los bosques secundarios son ecosistemas retomados después de un cambio, usualmente antrópico. En estos lugares, los efectos de las intervenciones como incendios o el uso de la tierra para actividades agropecuarias aún son reconocibles y tienen una diversidad menor a aquella de bosques primarios del mismo tipo (Ficetola et al., 2008). Estos bosques poseen árboles con muchas especies pioneras, con una diversidad menor de árboles. Considerando que los bosques primarios suelen ser más diversos que los bosques secundarios, ya que albergan mayor variedad de organismos (Gibson et al., 2011), esperábamos encontrar este patrón en nuestras comparaciones. Aunque encontramos una ligera mayor riqueza de especies en bosques primarios que secundarios según los análisis de rarefacción. Sin 


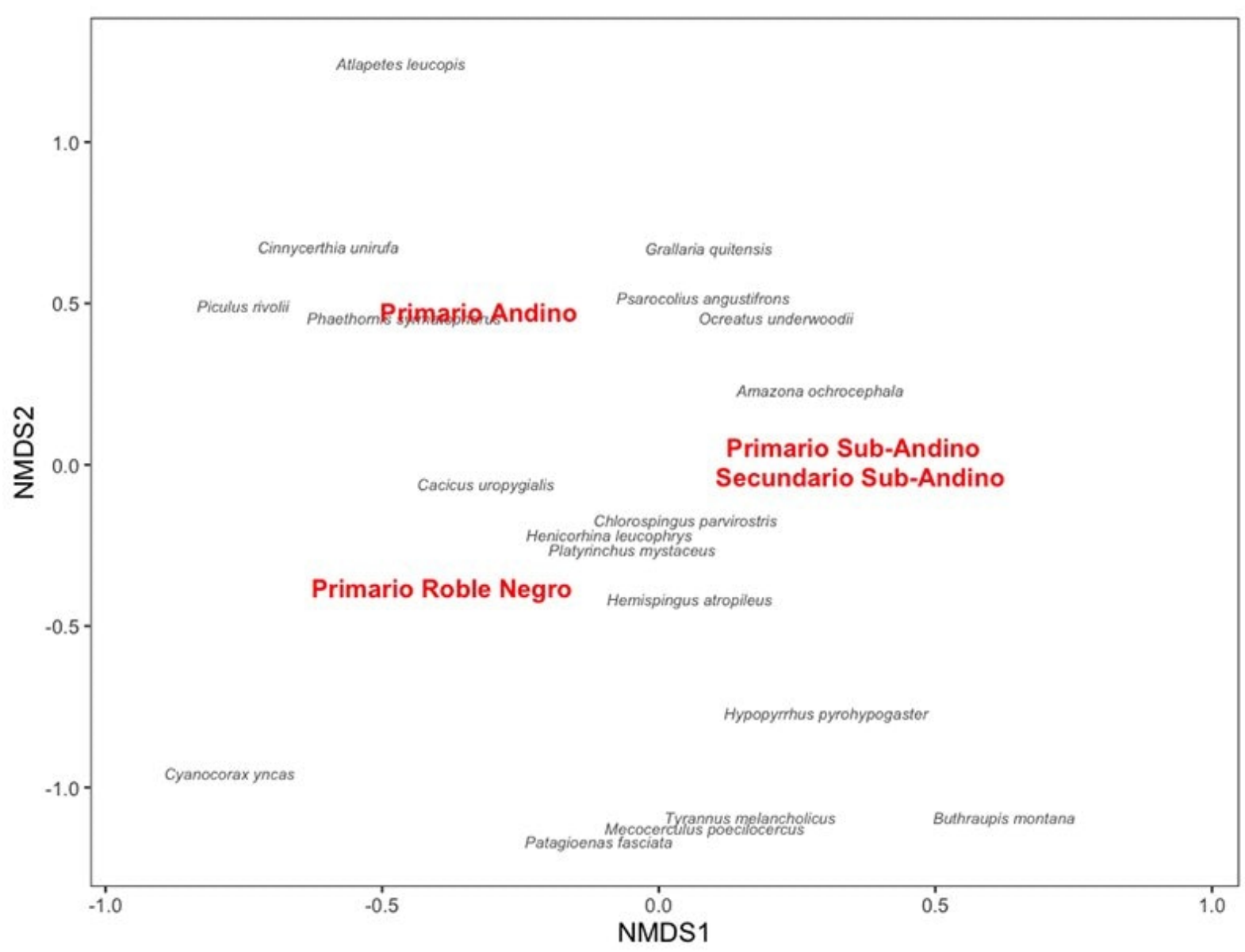

Figura 5. Ordenación con análisis de escalamiento multidimensional no paramétrico (NMSD) de las comunidades de aves en los cuatro tipos de bosque muestreados en el Parque Nacional Natural Cueva de Los Guacharos (rojo) y las especies más frecuentemente registradas (negro).

Tabla 1. Especies más abundantes registradas en las comunidades de aves, para cada tipo de bosque muestreado, en el Parque Nacional Natural Cueva de Los Guacharos

\begin{tabular}{|c|c|c|c|}
\hline Bosque Primario Andino & $\begin{array}{l}\text { Bosque Primario Roble Ne- } \\
\text { gro }\end{array}$ & $\begin{array}{lll}\text { Bosque } & \text { Primario } & \text { Sub- } \\
\text { Andino } & & \\
\end{array}$ & $\begin{array}{l}\text { Bosque Secundario Sub- } \\
\text { Andino }\end{array}$ \\
\hline Colaptes rivolii & Zimmerius chrysops & Henicorhina leucophrys & Henicorhina leucophrys \\
\hline Amblycercus holosericeus & Myioborus miniatus & Cyanocorax yncas & Rupicola peruvianus \\
\hline Catamblyrhynchus diadema & Euphonia laniirostris & Hypopyrrhus pyrohypogaster & Hypopyrrhus pyrohypogaster \\
\hline Chlorornis riefferii & Cyanocorax yncas & Rupicola peruvianus & Cyanocorax yncas \\
\hline Cinnycerthia unirufa & Patagioenas fasciata & Ocreatus underwoodii & Psarocolius angustifrons \\
\hline Mecocerculus poecilocercus & Mecocerculus poecilocercus & Myioborus miniatus & Tyrannus melancholicus \\
\hline Atlapetes leucopis & Nyctidromus albicollis & Phaethornis syrmatophorus & Amazona ochrocephala* \\
\hline Chlorospingus parvirostris & Amazona ochrocephala** & Arremon brunneinucha & Ocreatus underwoodii \\
\hline Grallaria quitensis** & Buthraupis montana & Zimmerius chrysops & Kleinothraupis atropileus \\
\hline Platyrinchus mystaceus & Cacicus uropygialis & Kleinothraupis atropileus & Cacicus uropygialis \\
\hline
\end{tabular}

(**) especie no confirmada según su distribución y que requiere más observaciones para confirmar su presencia. 
embargo, según el índice entrópico de Shannon, estas diferencias en diversidad no resultaron ser estadísticamente significativas, como se ha encontrado en otros estudios en bosques secundarios viejos (Borges, 2007). Este resultado es importante, dado que estos bosques secundarios se empezaron a formar desde comienzos de 1970 y en tres décadas y media lograron establecer unos ensamblajes de aves muy similares a las de bosques subandinos primarios mixtos. Esto no lo esperábamos dadas las diferencias en la composición florística, porque buena parte de los recursos que usan las aves son diferentes (Prada y Stevenson, 2016). Nuestros resultados sugieren una alta resiliencia de estas comunidades de aves de los bosques secundarios, donde predominaban los potreros antes de los años 1970's. Posiblemente, esta alta recuperación a su vez está relacionada con el hecho de que estos bosques han estado libres de grandes disturbios y protegidos de la cacería (Parques Nacionales Naturales, 2005). Por último, muchas de las plantas de bosques secundarios son dispersadas por aves (e.g. Chamaedorea linearis, Ch. pinnatifrons, Oreopanax microcephalus, Nectandra acutifolia y Prestoea acuminata) (Cruz-Tejada et al., 2018; Stevenson et al., 2017) y constituyen recursos importantes para muchos elementos de la avifauna. Adicionalmente, se resalta que todas las especies que tienen registros de algún grado de amenaza de extinción se observaron en estos bosques, indicando su importancia en la conservación de especies. Especies que son raras en altas elevaciones en montañas, como el águila harpía (Harpia harpyja), fueron registradas y observadas por dos de los coautores de este trabajo, lo que puede deberse a una densidad relativamente alta de micos churucos (ca. 20 individuos por $\mathrm{km}^{2}$, Vargas et al., 2014), que son presas importantes para las harpías.

Las comparaciones de la composición de aves por tipo de bosque también resaltaron las similitudes entre los bosques subandinos mixtos

Tabla 2. Especies con categoría de amenaza de la UICN registradas en las comunidades de aves del Parque Nacional Natural Cueva de Los Guácharos

\begin{tabular}{l|l|l|l}
\hline Nombre científico (Familia) & Tipo de Bosque & $\begin{array}{l}\text { Libro Rojo Aves de } \\
\text { Colombia }\end{array}$ & Red list UICN \\
\hline Tinamus osgoodi (Tinamidae) & BSP, BSS & EN & VU \\
Aburria aburri (Cracidae) & BPRN, BSP, BSS & NI & NT \\
Patagioenas subvinacea (Columbidae) & BSP, BSS & NI & VU \\
Anthocephala berlepschi (Trochilidae) & BPS, BSS & VU & VU \\
Harpia harpyja* (Accipitridae) & BSS & NT & NT \\
Accipiter poliogaster** (Accipitridae) & BSS & NI & NT \\
Hapalopsittaca amazonina (Psittacidae) & BSS & VU & VU \\
Ognorhynchus icterotis (Psittacidae) & BSS & EN & VU \\
Psittacara wagleri (Psittacidae) & BSS & NI & NT \\
Grallaricula cucullata (Grallariidae) & BSP, BSS & LC & VU \\
Pipreola chlorolepidota (Cotingidae) & BSS & NT & NT \\
Atlapetes fuscoolivaceus (Passerellidae) & BSP, BSS & VU & NT \\
Hypopyrrhus pyrohypogaster (Icteridae) & BSP, BSS & VU & VU \\
Sericossypha albocristata (Thraupidae) & BAP, BSP, BSS & NI & VU \\
Andigena nigrirostris (Ramphastidae) & BSP, BSS & NT & LC \\
Creurgops verticalis (Thraupidae) & BSS & VU & LC \\
Drymophila caudata (Thamnophilidae) & BSS & NI & NT \\
\hline
\end{tabular}

BAP: Bosque Primario Andino; BPRN: Bosque Primario de Roble Negro; BSP: Bosque Primario Sub-Andino; BSS: Bosque Secundario Sub- Andino.

NI: No incluido; LC: Preocupación menor; NT: Casi amenazada; VU: Vulnerable; EN: En peligro. (*) registro inusual pero confirmado; $\left(^{* *}\right)$ especie no confirmada según su distribución y que requiere más observaciones para confirmar su presencia. 
primarios y los secundarios. Según la ordenación, se evidencia claramente que las comunidades de aves son distintas en los bosques andinos, lo que puede deberse a la altura, temperatura y composición florística de los bosques. A nivel de composición de las comunidades de avifauna es también clara la diferencia observada en los bosques de roble negro. Estas diferencias no se pueden entender por aspectos climáticos, ya que estos bosques se encuentran en elevaciones de bosques subandinos y no se han encontrado parámetros ambientales que los diferencien fácilmente de los bosques subandinos mixtos (Prada y Stevenson, 2016). Por lo tanto, proponemos que sean comunidades de aves mayormente generalistas, que pueden encontrar recursos como insectos o flores $\mathrm{y}$ frutos de poca abundancia.

Concluimos que se encontró apoyo parcial a la principal hipótesis planteada, mostrando diferencias en composición de las comunidades de aves en distintos tipos de bosque (exceptuando los bosques primarios y secundarios subandinos) y cambios altitudinales asociados a una comunidad diferente en bosques andinos. Las diferencias parecen estar asociadas a varios factores, como la composición de plantas y abundancia de recursos (también contribuyendo a la separación de bosques andinos, pero principalmente a la discriminación de los bosques subandinos de roble negro) y la temperatura separando los bosques andinos de los otros. Pese a lo anterior, no está claro cómo las comunidades de bosques subandinos mixtos primarios y secundarios pueden tener unas características tan similares, a pesar de las diferencias florísticas. Es factible que, dada la movilidad de muchas de estas especies y la cercanía de estos tipos de bosques (figura 1), esto sea parte de la explicación. Por último, es posible que los tipos de bosques con menos especies corresponden a aquellos con condiciones climáticas más adversas y largos períodos de escasez de recursos (Wright et al., 1999); por lo tanto, los patrones de diversidad encontrados podrían estar relacionados con la hipótesis de mayor productividad conduciendo a un mayor número de especies y de mayor estrés ambiental (condiciones adversas), generando comunidades poco diversas.

\section{AGRADECIMIENTOS}

Agradecemos al exdirector del parque, señor Ítalo Rodríguez y a Alejandro Fonseca, por su colaboración para realizar este estudio. A Juan Alejandro Morales, Camila Gómez y Cristina Rueda por liderar las actividades de campo con estudiantes de la Universidad de Los Andes, quienes aportaron algunos registros a la lista de especies de la zona. A Carolina García y Sasha Cárdenas por la ayuda con el mapa y las figuras. A Camila Gómez por sus comentarios y a Ana María Martínez por sus aportes en las versiones iniciales del manuscrito. Finalmente, a la Universidad de los Andes y la Unidad de Parques Nacionales Naturales, por los permisos y la posibilidad de realizar actividades en el PNN Cueva de Los Guácharos.

\section{CONFLICTO DE INTERESES}

Los autores declaran que no tienen conflictos de interés relacionados con la publicación de es este manuscrito.

\section{REFERENCIAS}

Álvarez, M., Umaña, A. M., Mejía, G. D., Cajiao, J., von Hildebrand, P., \& Gast, F. (2003). Aves del Parque Nacional Natural Serranía de Chiribiquete, Amazonia-Provincia de la Guyana, Colombia. Biota Colombiana, 4(1), 49-63. https://www.redalyc.org/pdf/491/49140103.pdf

Armenteras, D., Rodríguez, N., Retana, J., \& Morales, M. (2011). Understanding deforestation in montane and lowland forests of the Colombian Andes. Regional Environmental Change, 11 (3), 693-705. DOI:10.1007/s10113010-0200-y

Ayerbe-Quiñones, F. (2018). Guía ilustrada de la avifauna colombiana. Wildlife Conservation Society.

Bojorges-Baños, J. C. B., \& López-Mata, L. L. (2006). Asociación de la riqueza y diversidad de especies de aves y estructura de la vegetación en una selva mediana subperennifolia en el centro de Veracruz, México. Revista Mexicana de Biodiversidad, 777(2), 235-249. https: //www.redalyc.org/pdf/425/42577210.pdf

Borges, S. H. (2007). Bird assemblages in secondary forests developing after slash-and-burn agriculture in the Brazilian Amazon. Journal of Tropical Ecology, 23(4), 469-477. DOI:10.1017/S0266467407004105

Cadena, C. D., Parra, J.L., Botero, C. A., Mejía, G. D., Aldana, J., \& Londoño, G. A. (1998). Bird species at the CIEM, Tinigua National Park: A partial list. Field Stud. Fauna Flora La Macarena Colombia. 12, 19-32. https://ci.nii.ac. 
jp/naid/10020148939/

Cadena, C. D., Pedraza, C. A., \& Brumfield, R. T. (2016). Climate, habitat associations and the potential distributions of Neotropical birds: Implications for diversification across the Andes. Revista de la Academia Colombiana de Ciencias Exactas, Físicas y Naturales, 40(155), 275-287. DOI:10.18257/raccefyn. 280

Cavelier, J., \& Goldstein, G. (1989). Mist and fog interception in elfin cloud forests in Colombia and Venezuela. Journal of Tropical Ecology, 5(3), 309-322. DOI:10.1017/S0266467400003709

Chapman, F. M. (1917). The distribution of bird life in Colombia, a contribution to a biological survey of South America. Bulletin of the American Museum of Natural History 36, 1-726. https://digitallibrary.amnh.org/handle/2246/ 1243

Cruz-Tejada, D. M., Acosta-Rojas, D. C., \& Stevenson, P. R. (2018). Are seeds able to germinate before fruit color ripening? Evidence from six Neotropical bird-dispersed plant species. Ecosphere, 9(6), e02174. DOI:10.1002/ecs2.2174

Etter, A., McAlpine, C., Wilson, K., Phinn, S., \& Possingham, H. (2006). Regional patterns of agricultural land use and deforestation in Colombia. Agriculture, Ecosystems \& Environment, 114(2-4), 369-386. DOI:10.1016/j.agee.2005.11.013

Ferreira, C. D., \& Perbiche-Neves, G. (2021). The importance of the standardizing sampling methodology to detect altitudinal gradients in mountains: A study case for the resident bird community in a hotspot (Atlantic forest) and the Middle Domain Effect. Acta Oecologica, 110, 103677. DOI:10.1016/j.actao.2020.103677

Ficetola, G. F., Furlani, D., Colombo, G., \& De Bernardi, F. (2008). Assessing the value of secondary forest for amphibians: Eleutherodactylus frogs in a gradient of forest alteration. Biodiversity and Conservation, 17(9), 21852195. DOI:10.1007/s10531-007-9280-5

Franco, P., Saavedra-Rodriguez, C. A., \& Kattan, G. H. (2007). Bird species diversity captured by protected areas in the Andes of Colombia: a gap analysis. Oryx, 41(1), 57-63. DOI:10.1017/S0030605306001372

Franco, A. M., Devenish, C., Barrero, M. C., \& Romero M. H. (2009). Colombia. En: C, Devenish, D. F, DíazFernández, R. P, Clay, I, Davidson, \& I, Yépez-Zabala (Eds.), Important Bird Areas Americas - Priority sites for biodiversity conservation (pp. 135 - 148). BirdLife International. http://datazone.birdlife.org/userfiles/file/ IBAs/AmCntryPDFs/Colombia.pdf

Gast, F., \& Stevenson, P. R. (2020). Abundancia relativa de mamíferos medianos y grandes en el Parque Nacional Cueva de Los Guácharos (Huila, Colombia). Biota Neotropica, 20(3), 7-11. DOI:10.1590/1676-0611-BN-20160305

Gibson, L., Lee, T. M., Koh, L. P., Brook, B. W., Gardner, T. A., Barlow, J., \& Sodhi, N. S. (2011). Primary forests are irreplaceable for sustaining tropical biodiversity. Nature, 478(7369), 378-381. DOI:10.1038/nature10425

Henao-Díaz, L. F., Pacheco-Fernández, N. M., ArgüelloBernal, S., Moreno-Arocha, M. M., \& Stevenson, P. R. (2012). Patrones de diversidad de epífitas en bosques de tierras bajas y subandinos. Colombia Forestal, 15(2),
161-172. http://www.scielo.org.co/scielo.php?script $=$ sci_arttext\&pid=S0120-07392012000200002\&lng =en

Kattan, G. H., Franco, P., Saavedra-Rodríguez, C. A., Valderrama, C., Rojas, V., Osorio, D., \& Martinez, J. (2006). Spatial components of bird diversity in the Andes of Colombia: implications for designing a regional reserve system. Conservation Biology, 20(4), 1203-1211. DOI:10.1111/j.1523-1739.2006.00402.x

Martínez, O., \& Rechberger, J. (2007). Características de la avifauna en un gradiente altitudinal de un bosque nublado andino en La Paz, Bolivia. Revista Peruana de Biología, 14(2), 225-236. http://www.scielo.org.pe/scielo.php?script $=$ sci arttext\&pid=S1727-99332007000300009\&lng=es.

Naranjo, L. G., Amaya, J. D., Eusse-González, D., CifuentesSarmiento Y. (2012). Guía de las Especies Migratorias de la Biodiversidad en Colombia. Aves. Vol. 1. Ministerio de Ambiente y Desarrollo Sostenible / WWF Colombia.

Oksanen, J., Blanchet, F. G., Kindt, R., Legendre, P., Minchin, P. R., O'hara, R. B., Simpson, G. L., Solymos, P., Stevens, M. H. M., Wagner, H., \& Oksanen, M. J. (2013). Package 'vegan'. Community ecology package, version, 2(9), 1-295. https://cran.r-project.org

Parques Nacionales Naturales (2005). Plan de manejo 2005-2009 Parque Nacional Natural Cueva de los Guácharos. https://www.parquesnacionales. gov.co/portal/wp-content/uploads/2020/10/ plan-de-manejo-pnn-cueva-de-los-guacharos.pdf

Prada, C. M., \& Stevenson, P. R. (2016). Plant composition associated with environmental gradients in tropical montane forests (Cueva de Los Guacharos National Park, Huila, Colombia). Biotropica, 48(5), 568-576. DOI:10.1111/btp.12331

R Development Core Team (2012). A language and environment for statistical computing. Vienna, Austria: $R$ Foundation for Statistical Computing; 2012. https://www. R-project.org.

Remsen, J. V., Jr., J. I. Areta, E. Bonaccorso, S. Claramunt, A. Jaramillo, D. F. Lane, J. F. Pacheco, M. B. Robbins, F. G. Stiles, \& K. J. Zimmer. Version (2021). A classification of the bird species of South America. American Ornithological Society. http://www.museum.lsu.edu/ Remsen/ SACCBaseline.htm

Renjifo, L. M., Servat, G. P., Goerck, J. M., Loiselle, B. A., \& Blake, J. G. (1997). Patterns of species composition and endemism in the northern Neotropics: a case for conservation of montane avifaunas. Ornithological Monographs, 48, 577-594. https://www.jstor.org/stable/40157554

Renjifo, L. M., Gómez, M. F., Tibatá, J. V., Villarreal, Á. M. A., Kattan, G. H., Espine, J. D. A., \& Girón, J. B. (2013). Libro rojo de aves de Colombia: Vol 1. Bosques húmedos de los Andes y Costa Pacífica. Editorial Pontificia Universidad Javeriana. http://hdl.handle.net/20.500.11761/ 31408

Stevenson, P. R., Cardona, L. M., Acosta-Rojas, D. C., HenaoDiaz, F., \& Cárdenas, S. (2017). Diet of oilbirds (Steatornis caripensis) in Cueva de los Guacharos National Park (Colombia): Temporal variation in fruit consumption, dispersal, and seed morphology. Ornitología Neotropical, 28, 295-307. https://journals.sfu.ca/ornneo/index.php/ 
ornneo/article/view/243

Suárez-Sanabria, N., \& Cadena, C. D. (2014). Diversidad y estructura de la avifauna del Valle de Lagunillas, Parque Nacional Natural El Cocuy, Colombia. Ornitología Colombiana, 14, 48-61. http:// asociacioncolombianadeornitologia.org/

Taki, H., Makihara, H., Matsumura, T., Hasegawa, M., Matsuura, T., Tanaka, H., Shun'ichi Makino, S., \& Okabe, K. (2013). Evaluation of secondary forests as alternative habitats to primary forests for flower-visiting insects. Journal of Insect Conservation, 17(3), 549-556. DOI:10.1007/s10841-012-9539-3

Tibshirani, R., \& Leisch, F. (2015). Functions for the Book "An Introduction to the Bootstrap". R package version. http://cran.r-project.org/web/packages/ bootstrap/bootstrap.pdf

Vargas, S. A., León, J., Ramírez, M., Galvis, N., Cifuentes, E., \& Stevenson, P. R. (2014). Population density and ecological traits of highland woolly monkeys at Cueva de los Guácharos National Park, Colombia. En: N. B. Grow, S. Gursky-Doyen, \& A. Krzton (Eds.), High altitude primates (pp. 85-102). Springer. DOI:10.1007/978-1-46148175-1_5

Vélez, D., Tamayo, E., Ayerbe-Quiñones, F., Torres, J., Rey, J., Castro-Moreno, C., Ramírez, B., \& Ochoa-Quintero, J. M. (2021). Distribution of birds in Colombia. Biodiversity Data Journal, 9, e59202. DOI:10.3897/BDJ.9.e59202

Wright, S. J., Carrasco, C., Calderon, O., \& Paton, S. (1999). The El Niño Southern Oscillation, variable fruit production, and famine in a tropical forest. Ecology, 80(5), 1632-1647. DOI:10.1890/00129658(1999)080[1632:TENOSO]2.0.CO;2 


\section{Anexos}

Anexo 1. Total de especies registradas en los muestreos realizados para el Parque Nacional Natural Cueva de los Guácharos, Huila (Colombia)

\begin{tabular}{|c|c|c|c|c|}
\hline Orden & Familia & Nombre común & Nombre científico & Autor \\
\hline \multirow{2}{*}{ Tinamiformes } & \multirow{2}{*}{ Tinamidae } & Tinamú de montaña & Nothocercus bonapartei & (G. R. Gray, 1867) \\
\hline & & Tinamú gigante & Tinamus osgoodi & (Conover, 1949) \\
\hline Anseriformes & Anatidae & Pato torrentoso & Merganetta armata & (Gould, 1842) \\
\hline \multirow{5}{*}{ Galliformes } & \multirow{5}{*}{ Cracidae } & Pava guacharaca & Chamaepetes goudotii & (Lesson, 1828) \\
\hline & & Pava de montaña & Penelope montagnii & (Bonaparte, 1856) \\
\hline & & Pava negra & Aburria aburri & (Lesson, 1828) \\
\hline & & Guacharaca & Ortalis columbiana & (Hellmayr, 1906) \\
\hline & & Perdiz colorada & Odontophorus hyperythrus & (Gould, 1858) \\
\hline \multirow{6}{*}{ Columbiformes } & \multirow{6}{*}{ Columbidae } & Torcaza collareja & Patagioenas fasciata & (Say, 1823) \\
\hline & & Torcaza morada & Patagioenas cayennensis & (Bonnaterre, 1792) \\
\hline & & Torcaza plomiza & Patagioenas plumbea & (Vieillot, 1818) \\
\hline & & Torcaza colorada & Patagioenas subvinacea & (Lawrence, 1868) \\
\hline & & Perdiz bigotuda & Zentrygon frenata & (Tschudi, 1843) \\
\hline & & Tortolita chusquera & Paraclaravis mondetoura & (Bonaparte, 1856) \\
\hline \multirow{3}{*}{ Cuculiformes } & \multirow{3}{*}{ Cuculidae } & Garrapatero común & Crotophaga ani & (Linnaeus, 1758) \\
\hline & & Cuco ardilla & Piaya cayana & (Linnaeus, 1766) \\
\hline & & Cuco migratorio & Coccyzus americanus & (Linnaeus, 1758) \\
\hline \multirow{2}{*}{ Caprimulgiformes } & \multirow{2}{*}{ Caprimulgidae } & Guardacamino de páramo & Systellura longirostris & (Bonaparte, 1825) \\
\hline & & Guardacaminos común & Nyctidromus albicollis & (Gmelin, 1789) \\
\hline \multirow{21}{*}{ Apodiformes } & \multirow{2}{*}{ Apodidae } & Vencejo de collar & Streptoprocne zonaris & (Shaw, 1796) \\
\hline & & Vencejo ceniciento & Chaetura cinereiventris & (P. L. Sclater, 1862) \\
\hline & \multirow{19}{*}{ Trochilidae } & Ermitaño leonado & Phaethornis syrmatophorus & (Gould, 1851) \\
\hline & & Chillón verde & Colibri cyanotus & (Swainson, 1827) \\
\hline & & Colibrí pechipunteado & Adelomyia melanogenys & (Fraser, 1840) \\
\hline & & Silfo coliverde & Aglaiocercus kingii & (Lesson, 1832) \\
\hline & & Cometa colinegro & Lesbia victoriae $^{* *}$ & $\begin{array}{l}\text { (Bourcier \& } \\
\text { Mulsant, 1846) }\end{array}$ \\
\hline & & Cometa coliverde & Lesbia nuna & (Lesson, 1832) \\
\hline & & Helechero común & Haplophaedia aureliae & $\begin{array}{l}\text { (Bourcier \& } \\
\text { Mulsant, 1846) }\end{array}$ \\
\hline & & Diminuto paramero & Eriocnemis aline & (Bourcier, 1842) \\
\hline & & Inca broncínea & Coeligena coeligena & (Lesson, 1833) \\
\hline & & Inca collareja & Coeligena torquata & (Boissonneau, 1840) \\
\hline & & Colibrí terciopelo & Lafresnaya lafresnayi & (Boissonneau, 1840) \\
\hline & & Colibrí chupasavia & Boissonneaua flavescens & (Loddiges, 1832) \\
\hline & & Cola de raqueta & Ocreatus underwoodii & (Lesson, 1832) \\
\hline & & Colibrí ventrirufo & Urosticte ruficrissa & (Lawrence, 1864) \\
\hline & & Heliodoxa coronada & Heliodoxa leadbeateri & (Bourcier, 1843) \\
\hline & & Colibrí esmeralda & Chlorostilbon gibsoni & (Fraser, 1840) \\
\hline & & Colibrí cabecicastaño & Anthocephala berlepschi & (Gould, 1854) \\
\hline & & Colibrí de bufón & Chalybura buffonii & (Lesson, 1832) \\
\hline & & Amazilia colirufo & Amazilia tzacatl & (De la Llave, 1833) \\
\hline
\end{tabular}




\begin{tabular}{|c|c|c|c|c|}
\hline Gruiformes & Rallidae & Chilacoa & Aramides cajaneus & (Statius Muller, 1776) \\
\hline \multirow{7}{*}{ Accipitriformes } & \multirow{7}{*}{ Accipitridae } & Águila harpía & Harpia harpyja* & (Linnaeus, 1758) \\
\hline & & Águila enmascarada & Spizaetus melanoleucus & (Vieillot, 1816) \\
\hline & & Gavilán milano gris & Ictinia plumbea & (J. F. Gmelin, 1788) \\
\hline & & Azor selvático & Accipiter poliogaster** & (Temminck, 1824) \\
\hline & & Azor cordillerano & Accipiter striatus & (Vieillot, 1808) \\
\hline & & Águila príncipe & Morphnarchus princeps & (Sclater, 1865) \\
\hline & & Gavilán negro & Parabuteo leucorrhous & (Quoy \& Gaimard, 1824) \\
\hline \multirow{4}{*}{ Trogoniformes } & \multirow{4}{*}{ Trogonidae } & Quetzal colinegro & Pharomachrus auriceps & (Gould, 1842) \\
\hline & & Quetzal crestado & Pharomachrus antisianus & (Orbigny, 1837) \\
\hline & & Trogón collarejo & Trogon collaris & (Vieillot, 1817) \\
\hline & & Trogón enmascarado & Trogon personatus & (Gould, 1842) \\
\hline Coraciiformes & Momotidae & Barranquero & Momotus aequatorialis & (Gould, 1858) \\
\hline \multirow{11}{*}{ Piciformes } & Capitonidae & Torito cabecirrojo & Eubucco bourcierii & (Lafresnaye, 1845) \\
\hline & \multirow{2}{*}{ Ramphastidae } & Tucancito Esmeralda & Aulacorhynchus prasinus & (Gould, 1833) \\
\hline & & Terlaque pechiazul & Andigena nigrirostris & (Waterhouse, 1839) \\
\hline & \multirow{8}{*}{ Picidae } & Carpintero oliváceo & Picumnus olivaceus & (Lafresnaye, 1845) \\
\hline & & Carpintero ahumado & Dryobates fumigatus & (Orbigny, 1840) \\
\hline & & Carpintero ventriamarillo & Dryobates dignus & $\begin{array}{l}\text { (P. L. Sclater \& Salvin, } \\
1877 \text { ) }\end{array}$ \\
\hline & & Carpintero gigante & Campephilus pollens & (Bonaparte, 1845) \\
\hline & & Carpintero marcial & Campephilus melanoleucos & (Gmelin, 1788) \\
\hline & & Carpintero cariblanco & Colaptes rubiginosus & (Swainson, 1820) \\
\hline & & Carpintero carmesí & Colaptes rivolii & (Boissonneau, 1840) \\
\hline & & Carpintero buchipecoso & Colaptes punctigula & (Boddaert, 1783) \\
\hline \multirow{4}{*}{ Falconiformes } & \multirow{4}{*}{ Falconidae } & Halcón rayado & Micrastur ruficollis & (Vieillot, 1817) \\
\hline & & Garrapatero & Milvago chimachima & (Vieillot, 1816) \\
\hline & & Halcón común & Falco sparverius & (Linnaeus, 1758) \\
\hline & & Gavilán golondrino & Falco rufigularis & (Daudin, 1800) \\
\hline \multirow{8}{*}{ Psittaciformes } & \multirow{8}{*}{ Psittacidae } & Lora carirroja & Hapalopsittaca amazonina & (Des Murs, 1845) \\
\hline & & Cotorra piquirroja & Pionus sordidus & (Linnaeus, 1758) \\
\hline & & Lora maicera & Pionus chalcopterus & (Fraser, 1841) \\
\hline & & Lora común & Amazona ochrocephala** & (Gmelin, 1788) \\
\hline & & Lora andina & Amazona mercenarius & (Tschudi, 1844) \\
\hline & & Perico colirrojo & Pyrrhura melanura & (Spix, 1824) \\
\hline & & Loro orejiamarillo & Ognorhynchus icterotis & (Massena \& Souance, 1854) \\
\hline & & Perico chocolero & Psittacara wagleri & (G. R. Gray, 1845) \\
\hline \multirow{11}{*}{ Passeriformes } & \multirow{4}{*}{ Thamnophilidae } & Hormiguerito rabibermejo & Euchrepomis callinota & (Sclater, 1855) \\
\hline & & Batará unicolor & Thamnophilus unicolor & (Sclater, 1859) \\
\hline & & Hormiguero rabilargo & Drymophila caudata & (Sclater, 1855) \\
\hline & & Hormiguero ojirrojo & Pyriglena maura & (Ménétries, 1835) \\
\hline & Conopophagidae & Zambullidor pechigris & Conopophaga castaneiceps & (Sclater, 1857) \\
\hline & \multirow{4}{*}{ Grallariidae } & Tororoi dorsiescamado & Grallaria guatimalensis & (Prevost \& DesMurs, 1846) \\
\hline & & Tororoi pechiblanco & Grallaria hypoleuca & (Sclater, 1855) \\
\hline & & Tororoi leonado & Grallaria quitensis** & (Lesson, 1844) \\
\hline & & Tororoi cabecirrufo & Grallaricula cucullata & (Sclater, 1856) \\
\hline & \multirow{2}{*}{ Rhinocryptidae } & Tapaculo negruzco & Scytalopus latrans & (Hellmayr, 1924) \\
\hline & & Churrín colilargo & Scytalopus micropterus & (Sclater, 1858) \\
\hline
\end{tabular}




\begin{tabular}{|c|c|c|c|}
\hline \multirow{16}{*}{ Furnariidae } & Trepador cordillero & Dendrocincla tyrannina & (Lafresnaye, 1851) \\
\hline & Trepador pico de cuña & Glyphorynchus spirurus & (Vieillot, 1819) \\
\hline & Trepador rayado & Dendrocolaptes picumnus & (Lichtenstein, 1820) \\
\hline & Trepador perlado & Xiphorhynchus triangularis & (Lafresnaye, 1842) \\
\hline & Guadañero estriado & Campylorhamphus pusillus & (P. L. Sclater, 1860) \\
\hline & Trepador montañero & Lepidocolaptes lacrymiger & (Lafresnaye, 1849) \\
\hline & Corretroncos cuellilargo & Pseudocolaptes boissonneautii & (Lafresnaye, 1840) \\
\hline & Corretroncos alirrufo & Premnornis guttuliger & (P. L. Sclater, 1864) \\
\hline & Hojarasquero montañero & Anabacerthia striaticollis & (Lafresnaye, 1841) \\
\hline & Hojarasquero listado & Syndactyla subalaris & (P. L. Sclater, 1859) \\
\hline & Hojarasquero menor & Thripadectes holostictus & $\begin{array}{l}\text { (P. L. Sclater \& } \\
\text { Salvin, 1876) }\end{array}$ \\
\hline & Corretroncos barranquero & Premnoplex brunnescens & (P. L. Sclater, 1856) \\
\hline & Corretroncos perlado & Margarornis squamiger & $\begin{array}{l}\text { (Orbigny \& Lafresna- } \\
\text { ye, 1838) }\end{array}$ \\
\hline & Rastrojero andino & Asthenes fuliginosa** & (Lafresnaye, 1843) \\
\hline & Rastrojero de azara & Synallaxis azarae & (Orbigny, 1835) \\
\hline & Saltarín moñudo & Masius chrysopterus & (Lafresnaye, 1843) \\
\hline \multirow{7}{*}{ Cotingidae } & Fruterito verdinegro & Pipreola riefferii & (Boissonneau, 1840) \\
\hline & Frutero pechinegro & Pipreola lubomirskii & (Taczanowski, 1879) \\
\hline & Frutero pigmeo & Pipreola chlorolepidota & (Swainson, 1838) \\
\hline & Gallito de roca & Rupicola peruvianus & (Latham, 1790) \\
\hline & Guardabosque oliva & Snowornis cryptolophus & $\begin{array}{l}\text { (P. L. Sclater \& } \\
\text { Salvin, 1877) }\end{array}$ \\
\hline & Cuaba & Querula purpurata & (Statius Muller, 1776) \\
\hline & Guardabosque cenizo & Lipaugus fuscocinereus & (Lafresnaye, 1843) \\
\hline Tityridae & Tityra enmascarada & Tityra semifasciata & (Spix, 1825) \\
\hline \multirow{14}{*}{ Tyrannidae } & Pico de pala crestiamarillo & Platyrinchus mystaceus & (Vieillot, 1818) \\
\hline & Tiranuelo colorado & Pseudotriccus ruficeps & (Lafresnaye, 1843) \\
\hline & Atrapamoscas pechirrufo & Leptopogon rufipectus & (Lafresnaye, 1846) \\
\hline & Tiranuelo pileado & Lophotriccus pileatus & (Tschudi, 1844) \\
\hline & Picochato carinegro & Hemitriccus granadensis & (Hartlaub, 1843) \\
\hline & Tiranuelo capirrufo & Poecilotriccus ruficeps & (Kaup, 1852) \\
\hline & Tiranuelo cejiamarillo & Zimmerius chrysops & (Sclater, 1859) \\
\hline & Tiranuelo coliblanco & Mecocerculus poecilocercus & $\begin{array}{l}\text { (P. L. Sclater \& } \\
\text { Salvin, 1873) }\end{array}$ \\
\hline & Tiranuelo gorgiblanco & Mecocerculus leucophrys & $\begin{array}{l}\text { (Orbigny \& Lafresna- } \\
\text { ye, 1837) }\end{array}$ \\
\hline & Sirirí común & Tyrannus melancholicus & (Vieillot, 1819) \\
\hline & Atrapamoscas capinegro & Myiarchus tuberculifer & $\begin{array}{l}\text { (Orbigny \& Lafresna- } \\
\text { ye, 1837) }\end{array}$ \\
\hline & Atrapamoscas montañero & Myiarchus cephalotes & (Taczanowski, 1880) \\
\hline & Pitajo diadema & Ochthoeca diadema & (Hartlaub, 1843) \\
\hline & Atrapamoscas guardapuente & Sayornis nigricans & (Swainson, 1827) \\
\hline \multirow{2}{*}{ Vireonidae } & Verderón cejirrufo & Cyclarhis gujanensis & (J. F. Gmelin, 1789) \\
\hline & Verderón ojirrojo & Vireo olivaceus & (Linnaeus, 1766) \\
\hline Corvidae & Quinquina urraca & Cyanocorax yncas & (Boddaert, 1783) \\
\hline Hirundinidae & Golondrina azul y blanca & Pygochelidon cyanoleuca & (Vieillot, 1817) \\
\hline
\end{tabular}




\begin{tabular}{|c|c|c|c|}
\hline \multirow{7}{*}{ Troglodytidae } & Cucarachero ruiseñor & Microcerculus marginatus & (Sclater, 1855) \\
\hline & Cucarachero común & Troglodytes aedon & (Vieillot, 1809) \\
\hline & Cucarachero montañero & Troglodytes solstitialis & (Sclater, 1859) \\
\hline & Cucarachero bigotudo & Pheugopedius mystacalis & (Sclater, 1860) \\
\hline & Cucarachero rufo & Cinnycerthia unirufa & (Lafresnaye, 1840) \\
\hline & Cucarachero sepia & Cinnycerthia olivascens & (Sharpe, 1881) \\
\hline & Cucarachero pechigris & Henicorhina leucophrys & (Tschudi, 1844) \\
\hline Cinclidae & Mirlo acuático & Cinclus leucocephalus & (Tschudi, 1844) \\
\hline \multirow{8}{*}{ Turdidae } & Solitario andino & Myadestes ralloides & (d’Orbigny, 1840) \\
\hline & Zorzal sombrío & Catharus fuscater & (Lafresnaye, 1845) \\
\hline & Zorzal pechiamarillo & Catharus maculatus & (Sclater, 1858) \\
\hline & Mirla ventriblanca & Turdus leucomelas & (Vieillot, 1818) \\
\hline & Mirla ollera & Turdus ignobilis & (Sclater, 1857) \\
\hline & Mirla colorada & Turdus fulviventris & (Sclater, 1857) \\
\hline & Mirla serrana & Turdus serranus & (Tschudi, 1844) \\
\hline & Mirla collareja & Turdus albicollis** & (Vieillot, 1818) \\
\hline \multirow{6}{*}{ Fringillidae } & Jilguero pechinegro & Spinus xanthogastrus & $\begin{array}{l}\text { (Du Bus de Gisignies, } \\
\text { 1855) }\end{array}$ \\
\hline & Jilguero aliblanco & Spinus psaltria & (Say, 1823) \\
\hline & Eufonia cabeciazul & Chlorophonia cyanocephala & (Vieillot, 1818) \\
\hline & Eufonia gorgiamarilla & Euphonia laniirostris & $\begin{array}{l}\text { (Orbigny \& Lafresna- } \\
\text { ye, 1837) }\end{array}$ \\
\hline & Eufonia común & Euphonia xanthogaster & (Sundevall, 1834) \\
\hline & Eufonia broncinea & Euphonia mesochrysa & (Salvadori, 1873) \\
\hline \multirow{6}{*}{ Passerellidae } & Montero bigotudo & Chlorospingus parvirostris & (Chapman, 1901) \\
\hline & Montero ojiblanco & Chlorospingus flavopectus & (Lafresnaye, 1840) \\
\hline & Atlapetes collarejo & Arremon brunneinucha & (Lafresnaye, 1839) \\
\hline & Copetón común & Zonotrichia capensis & (Statius Muller, 1776) \\
\hline & Gorrión sanjuanero & Atlapetes fuscoolivaceus & (Chapman, 1914) \\
\hline & Atlapetes pardusco & Atlapetes leucopis & $\begin{array}{l}\text { (P. L. Sclater \& } \\
\text { Salvin, 1878) }\end{array}$ \\
\hline \multirow{7}{*}{ Icteridae } & Arrendajo negro & Amblycercus holosericeus & (Deppe, 1830) \\
\hline & Oropéndola común & Psarocolius angustifrons & (Spix, 1824) \\
\hline & Arrendajo escarlata & Cacicus uropygialis & (Lafresnaye, 1843) \\
\hline & Arrendajo de montaña & Cacicus chrysonotus & (Bonaparte, 1845) \\
\hline & Turpial montañero & Icterus chrysater & (Lesson, 1844) \\
\hline & Chamón parasito & Molothrus bonariensis & (Gmelin, 1789) \\
\hline & Chango colombiano & Hypopyrrhus pyrohypogaster & (Tarragon, 1847) \\
\hline \multirow{6}{*}{ Parulidae } & Reinita tropical & Setophaga pitiayumi & (Vieillot, 1817) \\
\hline & Reinita naranja & Setophaga fusca & (Statius Muller, 1776) \\
\hline & Arañero coronado & Myiothlypis coronata & (Tschudi, 1844) \\
\hline & Arañero ribereño & Basileuterus tristriatus & (Tschudi, 1844) \\
\hline & Abanico pechinegro & Myioborus miniatus & (Swainson, 1827) \\
\hline & Abanico cariblanco & Myioborus ornatus & (Boissonneau, 1840) \\
\hline Cardinalidae & Piranga cabecirroja & Piranga rubriceps & (G. R. Gray, 1844) \\
\hline \multirow{3}{*}{ Thraupidae } & Pollo de monte & Sericossypha albocristata & (Lafresnaye, 1843) \\
\hline & Gorrión afelpado & Catamblyrhynchus diadema & (Lafresnaye, 1842) \\
\hline & Mielero opalino & Iridophanes pulcherrimus & (P. L. Sclater, 1853) \\
\hline
\end{tabular}




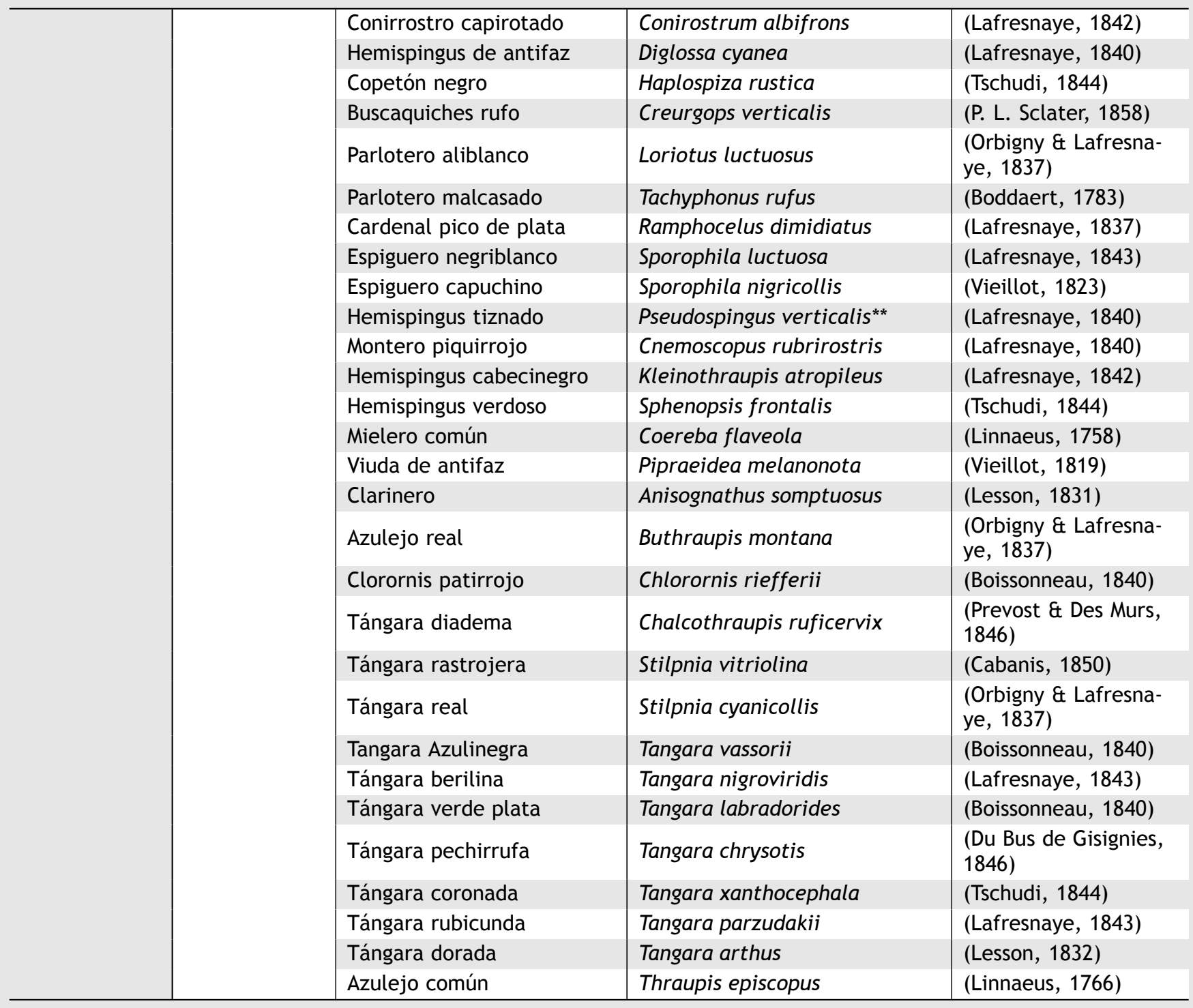

(*) registro inusual pero confirmado

(**) especie no confirmada según su distribución y que requiere más observaciones para confirmar su presencia 
Anexo 2. Especies migratorias registradas en las comunidades de aves del Parque Nacional Natural Cueva de Los Guácharos (de acuerdo con Naranjo et al. 2012)

\begin{tabular}{|c|c|c|c|c|c|c|c|}
\hline \multirow{2}{*}{ Especie } & \multicolumn{4}{|c|}{ Tipo de migración } & \multicolumn{3}{|c|}{ Estatus de residencia } \\
\hline & Lat & Alt & Trans & Loc & Inr & Irp & Rni \\
\hline Aburria aburri & & $x$ & & $x$ & & & $\mathrm{X}$ \\
\hline Accipiter poliogaster ${ }^{* *}$ & $x$ & & $x$ & & $x$ & & \\
\hline Aulacorhynchus prasinus & & $x$ & & $x$ & & & $\mathrm{X}$ \\
\hline Campylorhamphus pusillus & & $x$ & & $x$ & & & $\mathrm{x}$ \\
\hline Coccyzus americanus** & $\mathrm{x}$ & & $x$ & & & $x$ & \\
\hline Eubucco bourcierii & & $x$ & & $x$ & & & $\mathrm{x}$ \\
\hline Patagioenas fasciata & & $\mathrm{x}$ & & $\mathrm{x}$ & & & $\mathrm{x}$ \\
\hline Patagioenas subvinacea & & $x$ & & $x$ & & & $x$ \\
\hline Pharomachrus antisianus & & $x$ & & & & & $x$ \\
\hline Pygochelidon cyanoleuca** & $x$ & & $x$ & & & $x$ & \\
\hline Setophaga fusca* & $\mathrm{x}$ & & $x$ & & $x$ & & \\
\hline Setophaga pitiayumi* & $\mathrm{x}$ & $x$ & & & & & $\mathrm{x}$ \\
\hline Trogon collaris & & $x$ & & $x$ & & & $x$ \\
\hline Tyrannus melancholicus & & & & $x$ & & & $x$ \\
\hline Vireo olivaceus* & $x$ & & $x$ & & & $x$ & \\
\hline
\end{tabular}

(*): Migratorio boreal; $(* *)$ : Migratorio austral.

Tipo de Migración: Lat: Latitudinal; Alt: Altitudinal; Trans: Transnacional; Loc: Local.

Estatus de Residencia: Inr: Invernante no Reproductivo; Irp: Invernante Reproductivo con Poblaciones Permanentes; Rni: Migrante Local. 